\title{
Identification of medaka magnetoreceptor and cryptochromes
}

\author{
Yunzhi Wang, Jianbin Chen, Feng Zhu \& Yunhan Hong* \\ Department of Biological Sciences, National University of Singapore, Singapore 117543, Singapore
}

Received September 13,2016; accepted October 16, 2016; published online November 17, 2016

\begin{abstract}
Magnetoreception is a hallmark ability of animals for orientation and migration via sensing and utilizing geomagnetic fields. Magnetoreceptor (MagR) and cryptochromes (Cry) have recently been identified as the basis for magnetoreception in Drosophila. However, it has remained unknown whether MagR and Cry have conserved roles in diverse animals. Here we report the identification and expression of magr and cry genes in the fish medaka (Oryzias latipes). Cloning and sequencing identified a single magr gene, four cry genes and one cry-like gene in medaka. By sequence alignment, chromosomal synteny and gene structure analysis, medaka cry 2 and magr were found to be the orthologs of human Cry2 and Magr, with crylaa and crylab being coorthologs of human Cryl. Therefore, magr and cry 2 have remained as single copy genes, whereas cryl has undergone two rounds of gene duplication in medaka. Interestingly, magr and cry genes were detected in various stages throughout embryogenesis and displayed ubiquitous expression in adult organs rather than specific or preferential expression in neural organs such as brain and eye. Importantly, magr knockdown by morpholino did not produce visible abnormality in developing embryos, pointing to the possibility of producing viable magr knockouts in medaka as a vertebrate model for magnet biology.
\end{abstract}

magnetoreception, MagR, cryptochrome, magnetogenetics

Citation: $\quad$ Wang, Y., Chen, J., Zhu, F., and Hong, Y. (2017). Identification of medaka magnetoreceptor and cryptochromes. Sci China Life Sci 60, 271-278. doi: $10.1007 / \mathrm{s} 11427-016-0266-5$

\section{INTRODUCTION}

Dozens of experiments have now shown that diverse animal species, including bees, salmons, sea turtles and birds, have the ability to sense and utilize the Earth's magnetic field for orientation and migration over long distances (Wiltschko and Wiltschko, 1988; Wiltschko and Wiltschko, 2005). This ability is called magnetoreception (MR). However, the nature of MR still remains a contested topic. Several models have been proposed to illustrate its mechanism (Johnsen and Lohmann, 2008). Among these models, the most widely accepted one is the chemical compass model, which was first proposed by Schulten (Schulten and Weller, 1978; Mohseni et al., 2014), then later modified by others (Ritz et al., 2000; Möller et

*Corresponding author (email: dbshyh@nus.edu.sg) al., 2004; Maeda et al., 2008; Cai and Plenio, 2013). According to this model, cryptochromes (Cry), which belong to the DNA photolyase/cryptochrome family (Todo et al., 1996; Cashmore et al., 1999; Todo, 1999), play an essential role in MR. Cry was first found to be a crucial part of the circadian system and later reported as necessary for the magnetosensitive behavior in Drosophila (Gegear et al., 2008; Gegear et al., 2010). Recently, Xie and his colleagues have identified a crucial partner protein Drosophila CG8198, the homologue of bacterial iron-sulfur cluster assembly Iscal, complementary to Cry that enables polarity sensing of magnetic fields. They named it as magnetoreceptor (MagR) and proposed the Cry/MagR system, in which the protein complex of Cry and MagR mediates MR (Qin et al., 2016).

Besides its function in orientation and migration, MR may also contribute to biomedical research. For example, in 
neuroscience, magnetogenetics was recently reported as a promising noninvasive technique to control gene expression and cellular activity in vivo (Etoc et al., 2013; Etoc et al., 2015; Long et al., 2015; Stanley et al., 2015; Stanley et al., 2016; Wheeler et al., 2016). It shows many advantages over the currently widely used techniques such as deep-brain stimulation and optogenetics (Wichmann and Delong, 2006; Zhang et al., 2011), which require surgical implantation of a wire electrode or optical fiber (Kringelbach et al., 2007; Häusser, 2014). More importantly, the technique that combines the genetic targeting of MagR with remote magnetic stimulation has been reported (Long et al., 2015). The study of MR will provide a solid basis for this emerging and promising technique.

The medaka fish (Oryzias latipes) is an excellent vertebrate model for developmental biology and functional genomics. As a prelude to medaka magnet biology, our study aimed at the identification of magnetoreceptor and cryptochrome homologs in this organism. We identified a single magr gene, four cry genes and one cry-like gene. All these genes exhibited ubiquitous RNA expression throughout lifetime. Moreover, we provided evidence that morpholino-based magr gene knockdown did not affect medaka survival, pointing to the possibility to produce magr knockout animals in medaka as a vertebrate model for magnet biology.

\section{RESULTS}

\section{Identification of medaka magr and cry genes}

Using the published fruit fly MagR (Drosophila CG8198) sequence as the protein query to BLAST against National Center for Biotechnology Information (NCBI) non-redundant and Ensembl protein database, we identified one single medaka magr gene predicting a protein of 129 amino acid residues.
The putative amino acid sequence was aligned with MagR from other species, after which a phylogenetic tree was constructed (Figure 1A). The topology is similar to a tree constructed previously for zebrafish (Zhou et al., 2016). Protein sequence alignment revealed that medaka MagR has a high degree of identity with human (85\%) (Figure S1 in Supporting Information). Besides, medaka magr possesses a conserved exon/intron structure with human $M A G R$ (Figure 2A). More importantly, there is a clear syntenic relationship between medaka and human in the magr-bearing chromosomal region (Figure 3). Thus, medaka magr is orthologous to the human $M A G R$.

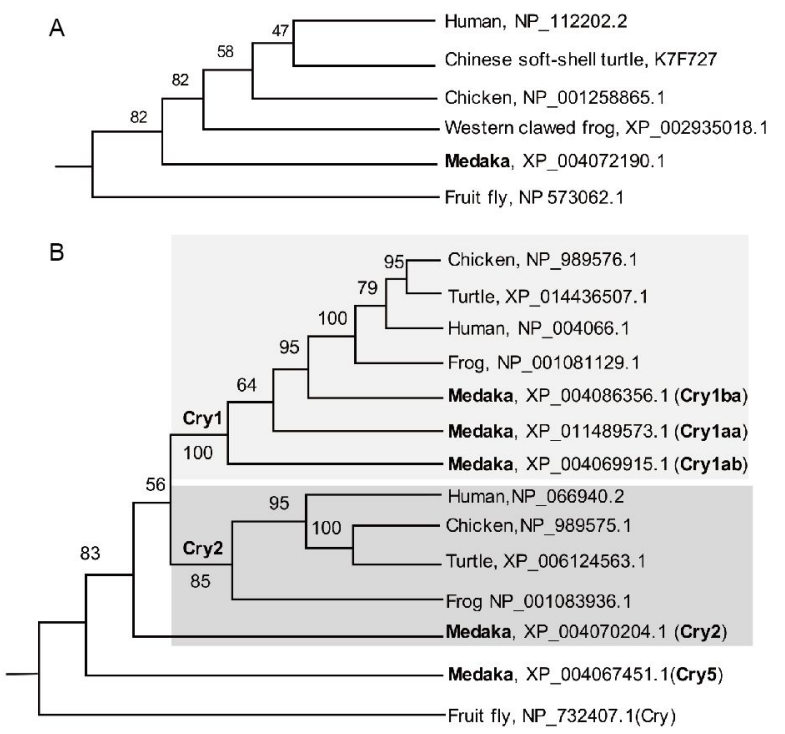

Figure 1 Phylogenetic tree by Neighbor-Jointing algorithm. A, MagR proteins. B, Cry proteins. Cry1 form one clade and Cry2 form another clade. Medaka Cry5 does not cluster with any other Cry proteins. Bootstrap values are given. Sequence accession numbers follow organisms. All medaka Cry proteins are shown in bold.

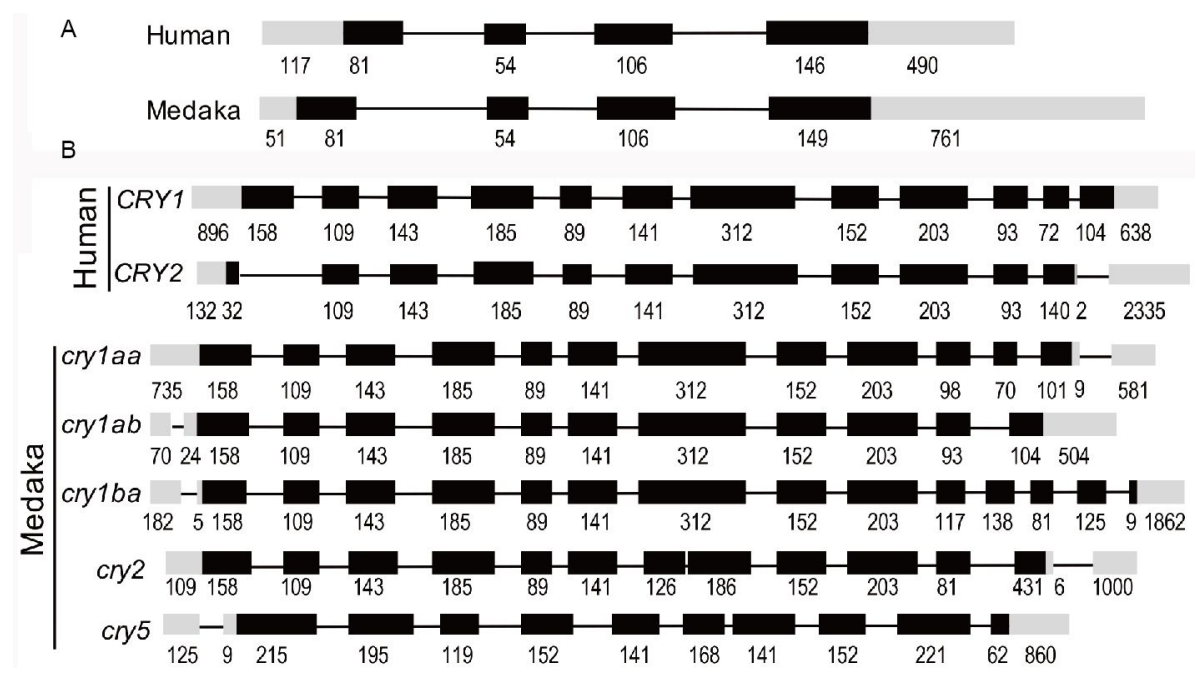

Figure 2 Gene structures of magr and cry in human and medaka. A, magr gene. B, cry genes. Exons are numbered with their sizes in bp. Notably, all cry genes except medaka cry5 have highly conserved exon-intron structures. Exons are not drawn to scale. Light grey column, UTR; black column, CDS; line, intron. 


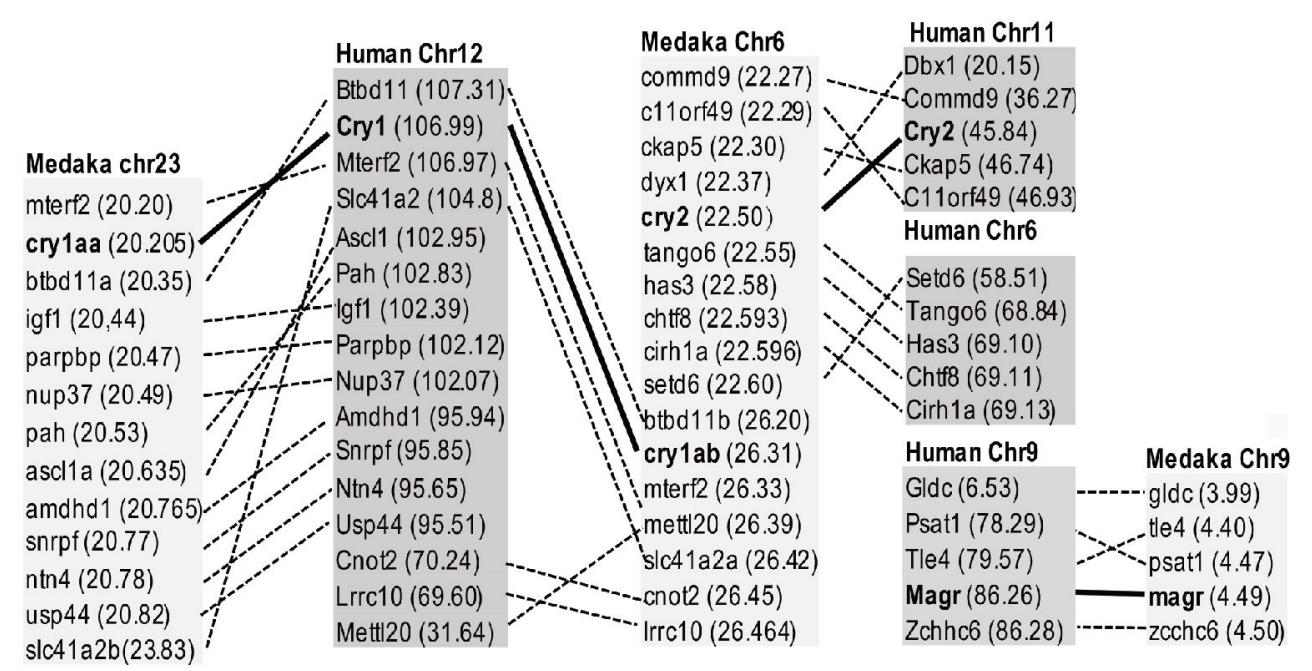

Figure 3 Chromosomal synteny of magr and cry genes. Shown are genes and chromosomal regions that clearly have syntenic relationships between medaka and human. Chromosomal positions are in parenthesis in million base pairs. Notably, crylab and cry 2 reside on the same chromosome, namely chromosome 6 , within a small region only $4 \mathrm{Mb}$ long in medaka.

Similarly, we also identified four medaka cry genes and one cry-like gene, namely crylaa, crylab, crylba, cry2 and cry5. By protein sequence alignment, medaka Crylaa, Crylab and Crylba displayed higher degrees of conservation with human CRY1 $(83 \%, 84 \%$ and $81 \%$, respectively) than human CRY2 (81\%, 80\% and 80\%, respectively); medaka Cry2 showed an identity of $75 \%$ to both human CRY1 and CRY2 (Figure S2 in Supporting Information). Phylogenetic analysis indicated that vertebrate Cry1 and Cry2 formed a sister clade (Figure 2A), implying they shared a common ancestry. By exon/intron structure analysis, conserved exon structures were found in medaka crylaa, crylab, crylba and human $C R Y 1$ and $C R Y 2$ with eight exons of the same length at the $5^{\prime}$ end (Figure 2B). In addition, medaka cry 2 also displayed a similar structure, with the only difference of one 312 bp exon splitting into two exons of 126 and 186 bp. Furthermore, comparisons of chromosome locations revealed clear syntenic relationships between the $c r y l$-bearing regions of human chromosome 12 and medaka chromosomes 6 and 23 , suggesting that the presence of crylaa and crylab in medaka fish was the consequence of whole genome duplication (Figure 3). In contrast, medaka cry5 gene was separated with others cry genes on the evolutionary trees. Besides, cry 5 exhibited an entirely different gene structure with other cry genes and no syntenic relationship was found between medaka cry 5 and human $C R Y$ genes. Moreover, crylba still has not been mapped on any chromosomes. Taken together, we could conclude that medaka has two cryl orthologs (crylaa and crylab) for human CRY1 and one cry2 ortholog for human $C R Y 2$.

\section{RNA expression pattern}

RT-PCR analysis was undertaken to examine cry and magr expression in developing embryos and adult organs (Figure 4). Embryos of nine different stages and 10 adult organs of ectoderm (brain, eye and skin), mesoderm (heart, kidney and muscle), endoderm (liver) and gonad (ovary and testis) were chosen. Interestingly, magr and cry were easily detected in early developing embryos before midblastula stage when bulk zygotic gene transcription takes place (Aizawa et al., 2003), demonstrating that they were maternally supplied. Furthermore, magr and cry genes were detected throughout embryogenesis and ubiquitously expressed in adult organs with differential expression patterns. Notably, magr and cry5 both exhibited a high level of RNA expression in ovary.

\section{magr is dispensable for organogenesis}

We analyzed the effect of magr depletion on medaka development by injection of MOmagr, a Morpholino oligo that targets the medaka magr mRNA, to one-cell stage embryos. When injected at $2 \mathrm{ng}$, there was no apparent difference in survival rates between morphant and wildtype (Table 1). Normal development was observed for most MOmagr injected embryos (Figure 5). A higher dose at $8 \mathrm{ng}$ didn't significantly increase the number of abnormally developing embryos and embryonic lethality, only leading to certain developmental retardation noticeable after epiboly (Figure S3 in Supporting Information), which might be the side effect of high dose morpholino injection. We chose seven marker genes for pluripotency and germ layers to examine their expressions in $2 \mathrm{ng}$ MOmagr knockdown and control embryos at different stages by RT-PCR (Figure 6). For the seven detected genes, namely nanog, oct4, cyt1, fgf8a, gata4, pax6a and otx2, no obvious differential expressions between knockdown and control groups were detected. Therefore, magr appears to be dispensable for medaka survival and organogenesis. 
Table 1 Development of MOmagr-injected medaka embryos ${ }^{\text {a) }}$

\begin{tabular}{|c|c|c|c|c|c|c|c|}
\hline \multirow{2}{*}{ Injection } & \multirow{2}{*}{ Embryo sample } & \multicolumn{6}{|c|}{ Embryos survived, $n(\%)^{*}$} \\
\hline & & $18 \mathrm{hpf}$ & $1 \mathrm{dpf}$ & $3 \mathrm{dpf}$ & $5 \mathrm{dpf}$ & Total fry & Normal fry ${ }^{* *}$ \\
\hline NO & 76 & 73(96) & 71(93) & $70(92)$ & $70(92)$ & $70(92)$ & $70(100)$ \\
\hline MOmagr & 80 & $72(90)$ & $72(90)$ & $70(88)$ & $69(86)$ & $68(85)$ & $65(96)$ \\
\hline
\end{tabular}

a) Embryos were non-injected or injected at the 1-cell stage with 2 ng of MOmagr and observed at indicated stage. hpf, hours post fertilization. dpf, days post fertilization. *, \% survival was obtained by comparisons between embryos sampled and embryos survived to stages of observation. **, $\%$ normal fry was obtained by a comparison between numbers of normal fry and total fry at hatching

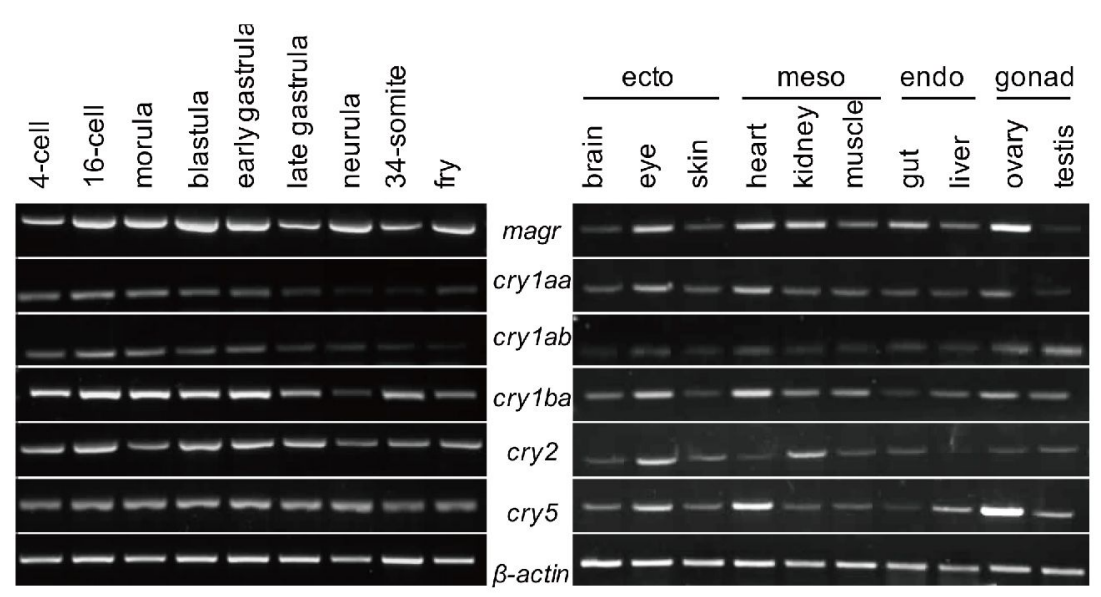

Figure 4 Expression of medaka magr and cry RNAs by RT-PCR. Left panel, Embryos at different stages. Right panel, Adult organs. magr and cry genes are maternally expressed and show a ubiquitous RNA expression in adult organs. ecto, ectoderm; meso, mesoderm; endo, endoderm.

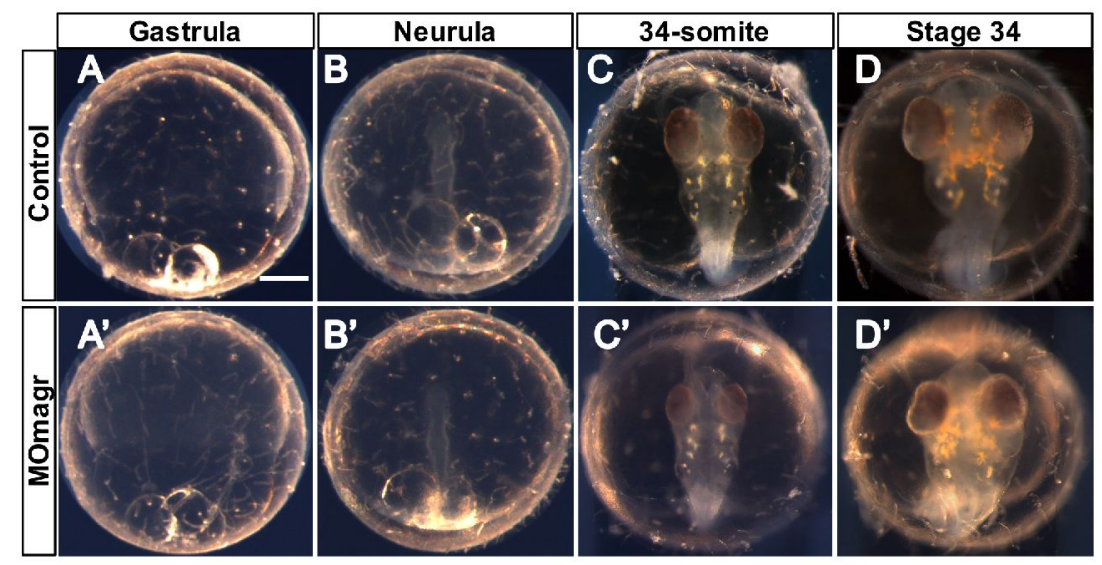

Figure 5 (Color online) magr knockdown by normal dose MOmagr has little effect on medaka organogenesis. Embryos were injected at the 1-cell stage with $2 \mathrm{ng}$ of MOmagr and phenotypically observed during embryogenesis. A-D, Non-injected control embryos. A'-D’, MOmagr-injected embryos. Scale bar 200 $\mu \mathrm{m}$.

\section{DISCUSSION}

The discovery of Cry/MagR magnetosensor system is a huge step towards fully understanding the molecular mechanism of animal MR. In this present study, we present preliminary data on the Cry/MagR system of medaka as a prelude to making use of this organism for magnet biology.

In medaka and other representative species we have chosen, we identified one single magr. Compared with the recent study on zebrafish magr, medaka MagR not only shows an high identity to human and zebrafish MagR (85\% and 87\%, respectively), but also displays the same exon/intron structure with human and zebrafish (Zhou et al., 2016), implying that magr may reserve its original function among these animals. Furthermore, we found four cry genes and one cry-like gene in medaka, indicating the gene duplication events and the divergent roles of these genes. The ancestral cry occurred likely before the first round of vertebrate genome duplication (Liu et al., 2015). Fish has undergone one additional whole genome duplication event after divergence from the tetrapod vertebrate $450 \mathrm{Ma}$ ago, leading to an additional duplication 


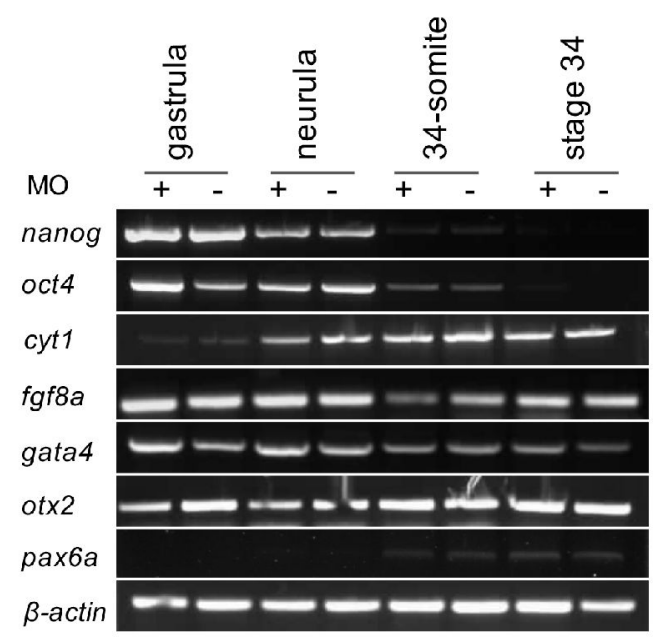

Figure 6 RT-PCR analysis of selected marker genes in MOmagr knockdown and control embryos. + ,embryos were injected at the 1-cell stage with 2 ng of MOmagr; -, non-injected embryos.

of genes (Amores et al., 1998). Based on their phylogenetic positions and their functions in regulating circadian rhythm, animal Cry proteins can be divided into two types (Yuan et al., 2007). One is mammalian-like Cry, or repressive Cry. This type of Cry can inhibit CLOCK:BMAL1-mediated transcription, functioning mainly as negative regulators of the clockwork's transcriptional feedback loop in a light-independent manner (Griffin et al., 1999; Kume et al., 1999). The other type is Drosophila-like Cry, or non-repressive Cry. This type of Cry does not inhibit CLOCK:BMAL1-mediated transcription, but functions instead as a light-dependent suppressor of PER/TIM-mediated inhibition of transcription (Ceriani et al., 1999). Former studies on zebrafish have shown that there are six Cry proteins, with Crylaa, Crylab, Crylba and Crylbb being mammalian-like, and Cry2 and Cry4 being Drosophila-like (Kobayashi et al., 2000; Liu et al., 2015; Zhou et al., 2016). The evolutionary relationship between zebrafish cry genes and other animals were also explored, and different nomenclature systems were introduced in the above studies. Based on the phylogeny, Huag et al. has also proposed their new nomenclature for cry recently (Haug et al, 2015). Our study follows Ensembl's nomenclature system and is consistent with Zhou's study (Zhou et al., 2016). Compared with zebrafish, medaka has only three cryl genes (crylaa, crylab, crylba) but no cry 4 gene. crylbb and cry 4 may be lost due to subsequent gene losses after the third round of teleost genome duplication (Liu et al., 2015). The topology of our evolutionary tree for cry is also similar to Zhou's result (Zhou et al., 2016). cryl genes and $c r y 2$ gene are clustered with $c r y 1$ genes and cry 2 genes from tetrapods, respectively. Besides, cry 5 appears to diverge earlier than cryl and cry 2 in the phylogenetic trees, and it has been reported to be clustered with 6-4 photolyases in previous phylogenetic studies (Oliveri et al., 2014), which can catalyze light-dependent DNA repair (Todo et al., 1993).
Therefore, these results indicate that medaka crylaa, crylab, crylba might be mammalian-like cry genes, and cry 2 might be Drosophila-like genes, whereas cry5 belongs to photolyase genes. Furthermore, it has been reported that human CRY2, pigeon Cry4, garden warbler Cry3 and cockroaches Cry2 are involved in MR (Liedvogel and Mouritsen, 2010; Foley et al., 2011; Bazalova et al., 2016; Qin et al., 2016). Specifically, pigeon Cry4 and human CRY2 have shown the ability to form complexes with MagR and can be co-purified (Qin et al., 2016). Hence, medaka cry2 might be a potential target for future medaka magnet biology study.

Further analysis by RT-PCR displayed that medaka magr and cry genes are maternally expressed, detectable throughout embryogenesis, and exhibited ubiquitous expression in adult organs rather than specific or preferential expression in neural organs such as brain and eye. A similar result was reported in zebrafish (Zhou et al., 2016). This implies the diverse roles of magr and cry. Unlike cry, which has already been reported to have other important functions such as circadian rhythm resetting and photosensitivity (Zhu et al., 2008; Chaves et al., 2011), the potential function of magr is to be explored. Since magr is the homologue of bacterial iron-sulfur cluster assembly Iscal, which is evolutionarily highly conserved and found in prokaryotic and eukaryotic organisms for the biogenesis of iron-sulfur cluster across species (Zheng et al., 1998; Schwartz et al., 2001; Vinella et al., 2009), it is predictable that magr may also have similar roles. For example, Nilsson et al. have reported that Iscal knockdown in zebrafish results in anemia (Nilsson et al., 2009). Moreover, the inhibition of magr expression in fruit fly has been reported to disrupt circadian behavior (Mandilaras and Missirlis, 2012). In medaka, we show that morpholino knockdown of magr did not affect survival rates of the medaka embryos at both 2 and 8 ng dose since most magr knockdown embryos survived and developed into fry. The retardation shown at $8 \mathrm{ng}$ dose could be the nonspecific effects of high dose morpholino injection (Heasman, 2002).

In summary, we identified magr and cry genes and found the orthologue of human MAGR, CRY1 and CRY2 genes in medaka. Besides, magr knockdown by morpholino did not produce visible abnormality in developing embryos. The meaning of our study is two-fold. On one hand, even though human are widely assumed not to have magnetic sense (Phillips et al., 2010), there is consistent evidence of the influence of geomagnetic field on the light sensitivity of human visual system (Thoss et al., 2000; Thoss et al., 2002). It has also been verified that human CRY2 has the molecular capability to function as a light-sensitive magnetosensor (Foley et al., 2011). Besides, Xie's group has reported the co-purification of MAGR and CRY2 in human (Qin et al., 2016). Therefore, using medaka as a vertebrate model for magnet biology will not only boost our knowledge of animal MR but also cast light on understanding human MR. On 
the other hand, magnetogenetics that combine the genetic targeting of MagR with remote magnetic stimulation has been developed as a promising method for non-invasive neuron stimulation, which might replace the invasive approaches such as optogenetics and deep-brain stimulation in neurobiology research (Long et al., 2015). Thus, our study of medaka magnet biology could provide a good model and help advance the technology of magnetogenetics.

\section{MATERIALS AND METHODS}

\section{Fish}

Work with medaka fish followed guidelines on the Care and Use of Animals for Scientific Purposes as outlined by the National Advisory Committee for Laboratory Animal Research in Singapore. Medaka strain Hd-rR was maintained under an artificial photoperiod of $14-\mathrm{h} / 10$-h light/darkness at $26^{\circ} \mathrm{C}$ as described (Hong et al., 2011). Embryogenesis was staged as described (Iwamatsu, 2004).

\section{Gene identification}

BLAST searches by using protein sequences of the fruit fly MagR and Cry as queries against the NCBI non-redundant and Ensembl protein database led to the identification of putative genes orthologous to magr and cry. Six representative species were interrogated for each gene, namely, fruit fly (Drosophila melanogaster), human (Homo sapiens), chicken (Gallus gallus), Chinese soft-shell turtle (Pelodiscus sinesis), medaka (Oryzias latipes) and African clawed frog (Xenopus laevis) for cry or western clawed frog (Xenopus tropicalis) for magr.

\section{Sequence Analysis}

Multiple sequence alignment was conducted by using the Vector NTI suite 11 (Invitrogen, USA). Phylogenetic trees were constructed by using the MEGA6 package from a matrix of pairwise genetic distances according to the neighbor-joining (NJ) algorithm, and 1,000 trials of bootstrap analyses were used to provide confidence estimates for tree topologies. Genomic organization and chromosomal locations were explored by comparing the cDNA and corresponding genomic sequences in NCBI and UCSC genome browser. SyMap 4.0 (Soderlund et al., 2011) and biomart function in Ensembl were used to determine the orthologues of medaka to human genes in the chromosomal regions flanking MAGR, CRY1, and CRY2.

\section{RNA isolation and RT-PCR analysis}

Total RNA from medaka embryos and adult organs was isolated by using the Trizol reagent (Invitrogen, USA). All organs were from 3-month-old adult female adult except for testis. Synthesis of cDNA templates was primed with oligo $(\mathrm{dT})_{25}$ by using M_MLC reverse transcriptase
(Promega, USA) (Hong et al., 2004). PCR was performed by using primers listed in Tables S1 and S2 in Supporting Information. $\beta$-actin was amplified from the same set of cDNA samples as an internal control. PCR was run in a $20-\mu \mathrm{L}$ volume containing $10 \mathrm{ng}$ of cDNA reaction for 26 cycles ( $\beta$-actin) and 30 cycles (magr, cry and other selected marker genes) of $30 \mathrm{~s}$ at $95^{\circ} \mathrm{C}, 10 \mathrm{~s}$ at $55^{\circ} \mathrm{C}$ and $60 \mathrm{~s}$ at $72^{\circ} \mathrm{C}$. The PCR products were separated on $1 \%$ agarose gels and documented with a bioimaging system (Synoptics, UK).

\section{Morpholino oligo}

MOmagr (5'-CGCTCGCACTAAGGAGGCAGACAT-3'), a Morpholino oligo designed to block the translation of medaka magr mRNA, was synthesized by GeneTools (Philomath, USA) and dissolved in water at $1 \mathrm{mmol} \mathrm{L}^{-1}$ as stock solution.

\section{Microinjection}

Medaka embryos at the one-cell cell stage were injected with 2 and 8 ng MOmagr per embryos. Injected embryos and control embryos without injection were reared in medaka embryo rearing medium and regularly observed for survival and phenotypes.

Compliance and ethics The author(s) declare that they have no conflict of interest.

Acknowledgements This work was supported by the National Research Foundation of Singapore (NRF-CRP7-2010-03).

Aizawa, K., Shimada, A., Naruse, K., Mitani, H., and Shima, A. (2003). The medaka midblastula transition as revealed by the expression of the paternal genome. Gene Expr Patterns 3, 43-47.

Amores, A., Force, A., Yan, Y.L., Joly, L., Amemiya, C., Fritz, A., Ho, R.K., Langeland, J., Prince, V., Wang, Y.L., Westerfield, M., Ekker, M., and Postlethwait, J.H. (1998). Zebrafish hox clusters and vertebrate genome evolution. Science 282, 1711-1714.

Bazalova, O., Kvicalova, M., Valkova, T., Slaby, P., Bartos, P., Netusil, R., Tomanova, K., Braeunig, P., Lee, H.J., Sauman, I., Damulewicz, M., Provaznik, J., Pokorny, R., Dolezel, D., and Vacha, M. (2016). Cryptochrome 2 mediates directional magnetoreception in cockroaches. Proc Natl Acad Sci USA 113, 1660-1665.

Cai, J., and Plenio, M.B. (2013). Chemical compass model for avian magnetoreception as a quantum coherent device. Phys Rev Lett 111, 2305031304.4143.

Cashmore, A.R., Jarillo, J.A., Wu, Y.J., and Liu, D. (1999). Cryptochromes: blue light receptors for plants and animals. Science 284, 760-765.

Ceriani, M.F., Darlington, T.K., Staknis, D., Más, P., Petti, A.A., Weitz, C.J., and Kay, S.A. (1999). Light-dependent sequestration of timeless by cryptochrome. Science 285, 553-556.

Chaves, I., Pokorny, R., Byrdin, M., Hoang, N., Ritz, T., Brettel, K., Essen, L.O., van der Horst, G.T.J., Batschauer, A., and Ahmad, M. (2011). The cryptochromes: blue light photoreceptors in plants and animals. Annu Rev Plant Biol 62, 335-364.

Etoc, F., Lisse, D., Bellaiche, Y., Piehler, J., Coppey, M., and Dahan, M. (2013). Subcellular control of Rac-GTPase signalling by magnetogenetic manipulation inside living cells. Nat Nanotech 8, 193-198.

Etoc, F., Vicario, C., Lisse, D., Siaugue, J.M., Piehler, J., Coppey, M., and Dahan, M. (2015). Magnetogenetic control of protein gradients inside 
living cells with high spatial and temporal resolution. Nano Lett 15 , 3487-3494.

Foley, L.E., Gegear, R.J., and Reppert, S.M. (2011). Human cryptochrome exhibits light-dependent magnetosensitivity. Nat Commun 2, 356.

Gegear, R.J., Casselman, A., Waddell, S., and Reppert, S.M. (2008). Cryptochrome mediates light-dependent magnetosensitivity in Drosophila. Nature 454, 1014-1018.

Gegear, R.J., Foley, L.E., Casselman, A., and Reppert, S.M. (2010). Animal cryptochromes mediate magnetoreception by an unconventional photochemical mechanism. Nature 463, 804-807.

Griffin, E.A., Staknis, D. and Weitz, C.J. (1999). Light-independent role of CRY1 and CRY2 in the mammalian circadian clock. Science 286, 768-771.

Haug, M.F., Gesemann, M., Lazović, V., and Neuhauss, S.C.F. (2015). Eumetazoan cryptochrome phylogeny and evolution. Genome Biol Evol 7, 601-619.

Häusser, M. (2014). Optogenetics: the age of light. Nat Meth 11, $1012-1014$.

Heasman, J. (2002). Morpholino oligos: making sense of antisense? Dev Biol 243, 209-214.

Hong, N., Li, Z. and Hong, Y. (2011). Fish stem cell cultures. Int J Biol Sci 7, 392-402.

Hong, Y., Liu, T., Zhao, H., Xu, H., Wang, W., Liu, R., Chen, T., Deng, J., and Gui, J. (2004). Establishment of a normal medakafish spermatogonial cell line capable of sperm production in vitro. Proc Natl Acad Sci USA 101, 8011-8016.

Iwamatsu, T. (2004). Stages of normal development in the medaka Oryzias latipes. Mechan Dev 121, 605-618.

Johnsen, S., and Lohmann, K.J. (2008). Magnetoreception in animals feature article. Physics Today 61, 29.

Kobayashi, Y., Ishikawa, T., Hirayama, J., Daiyasu, H., Kanai, S., Toh, H., Fukuda, I., Tsujimura, T., Terada, N., Kamei, Y., Yuba, S., Iwai, S., and Todo, T. (2000). Molecular analysis of zebrafish photolyase/cryptochrome family: two types of cryptochromes present in zebrafish. Genes Cells 5, 725-738.

Kringelbach, M.L., Jenkinson, N., Owen, S.L.F., and Aziz, T.Z. (2007). Translational principles of deep brain stimulation. Nat Rev Neurosci 8, 623-635.

Kume, K., Zylka, M.J., Sriram, S., Shearman, L.P., Weaver, D.R., Jin, X., Maywood, E.S., Hastings, M.H., and Reppert, S.M. (1999). mCRY1 and mCRY2 are essential components of the negative limb of the circadian clock feedback loop. Cell 98, 193-205.

Liedvogel, M., and Mouritsen, H. (2010). Cryptochromes-a potential magnetoreceptor: what do we know and what do we want to know? J R Soc Interface 7, S147-S162.

Liu, C., Hu, J., Qu, C., Wang, L., Huang, G., Niu, P., Zhong, Z., Hong, F., Wang, G., Postlethwait, J.H., and Wang, H. (2015). Molecular evolution and functional divergence of zebrafish (Danio rerio) cryptochrome genes. Sci Rep 5, 8113.

Long, X., Ye, J., Zhao, D., and Zhang, S.J. (2015). Magnetogenetics: remote non-invasive magnetic activation of neuronal activity with a magnetoreceptor. Sci Bull 60, 2107-2119.

Möller, A., Sagasser, S., Wiltschko, W., and Schierwater, B. (2004). Retinal cryptochrome in a migratory passerine bird: a possible transducer for the avian magnetic compass. Naturwissenschaften 91, 585-588.

Maeda, K., Henbest, K.B., Cintolesi, F., Kuprov, I., Rodgers, C.T., Liddell, P.A., Gust, D., Timmel, C.R., and Hore, P.J. (2008). Chemical compass model of avian magnetoreception. Nature 453, 387-390.

Mandilaras, K., and Missirlis, F. (2012). Genes for iron metabolism influence circadian rhythms in Drosophila melanogaster. Metallomics 4, 928-936.

Mohseni, M., Omar, Y., Engel, G.S. and Plenio, M.B. (2014). Quantum Effects in Biology. (Cambridge: Cambridge University Press).

Nilsson, R., Schultz, I.J., Pierce, E.L., Soltis, K.A., Naranuntarat, A., Ward, D.M., Baughman, J.M., Paradkar, P.N., Kingsley, P.D., Culotta, V.C., Kaplan, J., Palis, J., Paw, B.H., and Mootha, V.K. (2009). Discovery of genes essential for heme biosynthesis through large-scale gene expression analysis. Cell Metab 10, 119-130.

Oliveri, P., Fortunato, A.E., Petrone, L., Ishikawa-Fujiwara, T., Kobayashi, Y., Todo, T., Antonova, O., Arboleda, E., Zantke, J., Tessmar-Raible, K., and Falciatore, A. (2014). The cryptochrome/photolyase family in aquatic organisms. Mar Genomics 14, 23-37.

Phillips, J.B., Muheim, R., and Jorge, P.E. (2010). A behavioral perspective on the biophysics of the light-dependent magnetic compass: a link between directional and spatial perception? J Exp Biol 213, 3247-3255.

Qin, S., Yin, H., Yang, C., Dou, Y., Liu, Z., Zhang, P., Yu, H., Huang, Y., Feng, J., Hao, J., Hao, J., Deng, L., Yan, X., Dong, X., Zhao, Z., Jiang, T., Wang, H.W., Luo, S.J., and Xie, C. (2016). A magnetic protein biocompass. Nat Mater 15, 217-226.

Ritz, T., Adem, S., and Schulten, K. (2000). A model for photoreceptorbased magnetoreception in birds. Biophysical J 78, 707-718.

Schulten, K., and Weller, A. (1978). Exploring fast electron transfer processes by magnetic fields. Biophys J 24, 295-305.

Schwartz, C.J., Giel, J.L., Patschkowski, T., Luther, C., Ruzicka, F.J., Beinert, H., and Kiley, P.J. (2001). IscR, an Fe-S cluster-containing transcription factor, represses expression of Escherichia coli genes encoding Fe-S cluster assembly proteins. Proc Natl Acad Sci USA 98, 14895-14900.

Soderlund, C., Bomhoff, M., and Nelson, W.M. (2011). SyMAP v3.4: a turnkey synteny system with application to plant genomes. Nucleic Acids Res 39, e68-e68.

Stanley, S.A., Kelly, L., Latcha, K.N., Schmidt, S.F., Yu, X., Nectow, A.R., Sauer, J., Dyke, J.P., Dordick, J.S., and Friedman, J.M. (2016). Bidirectional electromagnetic control of the hypothalamus regulates feeding and metabolism. Nature 531, 647-650.

Stanley, S.A., Sauer, J., Kane, R.S., Dordick, J.S., and Friedman, J.M. (2015). Remote regulation of glucose homeostasis in mice using genetically encoded nanoparticles. Nat Med 21, 92-98.

Thoss, F., Bartsch, B., Fritzsche, B., Tellschaft, D., and Thoss, M. (2000). The magnetic field sensitivity of the human visual system shows resonance and compass characteristic. J Comp Physiol A 186, 1007-1010.

Thoss, F., Bartsch, B., Tellschaft, D., and Thoss, M. (2002). The light sensitivity of the human visual system depends on the direction of view. J Comp Physiol A 188, 235-237.

Todo, T. (1999). Functional diversity of the DNA photolyase/blue light receptor family. Mutation Res/DNA Repair 434, 89-97.

Todo, T., Ryo, H., Yamamoto, K., Toh, H., Inui, T., Ayaki, H., Nomura, T., and Ikenaga, M. (1996). Similarity among the Drosophila (6-4)photolyase, a human photolyase homolog, and the DNA photolyase-bluelight photoreceptor family. Science 272, 109-112.

Todo, T., Takemori, H., Ryo, H., Ihara, M., Matsunaga, T., Nikaido, O., Sato, K., and Nomura, T. (1993). A new photoreactivating enzyme that specifically repairs ultraviolet light-induced (6-4)photoproducts. Nature 361, 371-374.

Vinella, D., Brochier-Armanet, C., Loiseau, L., Talla, E., and Barras, F. (2009). Iron-sulfur $(\mathrm{Fe} / \mathrm{S})$ protein biogenesis: phylogenomic and genetic studies of A-Type carriers. PLoS Genet 5, e1000497.

Wheeler, M.A., Smith, C.J., Ottolini, M., Barker, B.S., Purohit, A.M., Grippo, R.M., Gaykema, R.P., Spano, A.J., Beenhakker, M.P., Kucenas, S., Patel, M.K., Deppmann, C.D., and Güler, A.D. (2016). Genetically targeted magnetic control of the nervous system. Nat Neurosci 19, 756-761.

Wichmann, T., and Delong, M.R. (2006). Deep brain stimulation for neurologic and neuropsychiatric disorders. Neuron 52, 197-204.

Wiltschko, W., and Wiltschko, R. (1988). Magnetic orientation in birds. In: R.F. Johnston, ed. Current Ornithology. (Berlin: Springer), pp. 67-121.

Wiltschko, W., and Wiltschko, R. (2005). Magnetic orientation and magnetoreception in birds and other animals. J Comp Physiol A 191, 675-693.

Yuan, Q., Metterville, D., Briscoe, A.D., and Reppert, S.M. (2007). Insect cryptochromes: gene duplication and loss define diverse ways to construct insect circadian clocks. Mol Biol Evol 24, 948-955. 
Zhang, F., Vierock, J., Yizhar, O., Fenno, L.E., Tsunoda, S., Kianianmomeni, A., Prigge, M., Berndt, A., Cushman, J., Polle, J., Magnuson, J., Hegemann, P., and Deisseroth, K. (2011). The microbial opsin family of optogenetic tools. Cell 147, 1446-1457.

Zheng, L., Cash, V.L., Flint, D.H. and Dean, D.R. (1998). Assembly of iron-sulfur clusters. Identification of an iscSUA-hscBA-fdx gene cluster from Azotobacter vinelandii. J Biol Chem 273, 13264-13272.
Zhou, Z., Peng, X., Chen, J., Wu, X., Wang, Y., and Hong, Y. (2016). Identification of zebrafish magnetoreceptor and cryptochrome homologs. Sci China Life Sci doi: 10.1007/s11427-016-0195-x.

Zhu, H., Sauman, I., Yuan, Q., Casselman, A., Emery-Le, M., Emery, P., and Reppert, S.M. (2008). Cryptochromes define a novel circadian clock mechanism in monarch butterflies that may underlie sun compass navigation. PLoS Biol 6, e4.

Open Access This article is distributed under the terms of the Creative Commons Attribution License which permits any use, distribution, and reproduction in any medium, provided the original author(s) and source are credited.

\section{SUPPORTING INFORMATION}

Figure S1 Sequence alignment of MagR from six representative species.

Figure S2 Sequence alignment of Cryptochromes (Crys) from six representative species.

Figure S3 magr knockdown by high dose MOmagr has little effect on medaka organogenesis.

Table S1 Primers used for RT-PCR analysis

Table S2 Primers used for MOmagr knockdown RT-PCR analysis

The supporting information is available online at life.scichina.com and www.springerlink.com. The supporting materials are published as submitted, without typesetting or editing. The responsibility for scientific accuracy and content remains entirely with the authors. 